\title{
THERMAL EFFECT ON FREE VIBRATION AND BUCKLING OF A DOUBLE-MICROBEAM SYSTEM
}

\author{
UDC 620.179.13+620.174]:534.1
}

\author{
Marija Stamenković Atanasov ${ }^{1}$, Danilo Karličić ${ }^{1}$, Predrag Kozić², \\ Goran Janevski ${ }^{2}$ \\ ${ }^{1}$ Mathematical Institute of the Serbian Academy of Sciences and Arts, Serbia \\ ${ }^{2}$ University of Niš, Department of Mechanical Engineering, Serbia
}

\begin{abstract}
The paper investigates the problem of free vibration and buckling of an EulerBernoulli double-microbeam system (EBDMBS) under the compressive axial loading with a temperature change effect. The system is composed of two identical, parallel simplysupported microbeams which are continuously joined by the Pasternak's elastic layer. Analytical expressions for the critical buckling load, critical buckling temperature, natural frequencies and frequencies of transverse vibration of the EBDMBS represented by the ratios are derived and validated by the results found in the literature. Also analytical expressions are obtained for various buckling states and vibration-phase of the EBDMBS. The temperature change effect is assumed to have an influence on both the microbeams. The length scale parameter, temperature change effect, critical buckling load, thickness/material parameter, Pasternak's parameter and Poisson's effect are discussed in detail. Also, as a clearer display of the thermo-mechanical response of EBDMBS, the paper introduces a critical scale load ratio of the modified and the local critical buckling loads in lowtemperature environs. Numerical results show that the critical buckling temperatures for classical theories are always higher than the critical buckling temperature for MCST systems.
\end{abstract}

Key Words: Thermal Effect, Double-microbeam System, Critical Buckling Load, Pasternak's Parameter, Poisson's Effect

\section{INTRODUCTION}

Micro and nano structures became an object of interest in modern science and technology just after their invention. They possess important mechanical, electrical and thermal performances that are higher than conventional structural materials. Using micro/nano

Received November 15, 2016 / Accepted February 23, 2017

Corresponding author: Goran Janevski

University of Niš, Department of Mechanical Engineering, A. Medvedeva 14, 18000 Nis, Serbia

E-mail: gocky.jane@gmail.com 
structures in a high temperature environment leads to certain changes in stiffness. Recently, the vibration and buckling studies of beams with the microstructure effect have been increasingly present in the scientific community. Researchers are motivated to develop theories such as the modified couple stress theory (MCST) which contains the material length scale parameter; also, they are able to describe size effects on the micro-scale. On the other hand, the classical continuum mechanics theories neither contain any internal material length scale parameter nor are they able to describe these effects. The structural elements such as beams, plates, and membranes in the micro or nano length scale are frequently used as components in micro/nano electromechanical systems (MEMS/NEMS).

With the rapid development of technology, functionally graded (FG) beams and plates are often used in MEMS/NEMS, such as the components in the shape of memory thin films alloy with a global thickness in the micro/nano scale, atomic force microscopes (AFMs), and electrically actuated MEMS devices [1-8]. As opposed to the strain theories which were introduced by Mindlin and Eshel [9], with five constants besides the Lamé constants, Lam et al. [1] presented a modified theory consisting of only three non-classical constants. Wang et al. [10] used the above theory to analyze the behavior of micro beams considering Euler-Bernoulli and Timoshenko beam theories. Analysis of bending and buckling of a thin beam were presented by Lazopoulos and Lazopoulos [11]. These results imply that the gradient coefficient has a significant effect on the buckling load while the surface effect of the energy is negligible. The modified couple stress theory has been used by many authors just as it has been mostly applied to micro beams. The non-local Bernoulli-Euler beam model was proposed by Peddieson et al. [12], using a constitutive equation after Eringen et al. [13] which contains two additional material constants. The non-local theories for the Bernoulli-Euler, Timoshenko, Reddy, and Levinson beams were developed by Reddy [14] in a unified way using the Hamilton principle and the non-local constitutive relation of Eringen et al. [13]. Park and Gao [18] used a modified couple stress theory with an Euler-Bernoulli formulation for the bending analysis of cantilever beams. Ma et al. [19] and Reddy et al. [17] developed a modified Timoshenko beam theory and investigation of the bending and free vibration of simplysupported beams using the Navier solution process and finite elements method.

The buckling analysis of functionally graded micro beams based on the MCST is presented in Nateghi et al. [20]. The free vibration of a single-layered graphene sheet resting on an elastic matrix as a Pasternak foundation model explored by using the modified couple stress theory is presented by Bekir and Civalek [21]. M. Simsek and Reddy [22] developed a united higher order beam theory for a functionally graded (FG) microbeam embedded in an elastic Pasternak medium using the modified couple stress theory. In the paper of Hendou and Mohammadi [23], an Euler-Bernoulli model has been used for vibration analysis of micro-beams with large transverse deflection where thermoelastic damping is considered to be the main damping mechanism and displayed as imaginary stiffness into the equation of motion by evaluating the temperature profile as a function of lateral displacement. Free vibration and buckling of microbeams with the temperature change effect is presented by Ke et al. [24]. The finding that the thermal effect on the fundamental frequency and critical buckling load is very low when the thickness of the microbeam has a similar value to that of the material length scale parameter and that it becomes significant when the thickness of the microbeam becomes larger, was of particular interest, and the present paper considers what may happen with a double microbeam system.

Scientists are trying to comprehend the vibration behavior of micro/nano structures with the effect of temperature changes. Primarily motivated by the last few studies [22, 
23, 24], this paper analyzes the free vibration and buckling behavior with the temperature change effect of the Euler-Bernoulli double-microbeam system (EBDMBS). To solve the higher-order main equations of the EBDMBS we use the Bernoulli-Fourier method. The system is composed of two identical and parallel, simply-supported beams which are continuously joined by the Pasternak's elastic layer. It is assumed that the temperature change effect has an impact on both the microbeams. The length scale parameter, temperature change effect, critical buckling load, thickness/material parameter, Pasternak's parameter and Poisson's effect are discussed in detail. The paper presents the impact of different above-mentioned parameters on the natural frequency, frequency under compressive axial loading, critical buckling load and critical temperature of EBDMBS with thermal effect. Also, results for various buckling state and vibration-phase of the EBDMBS are obtained. The vibration phases include out-of-phase and in-phase modes of vibration. In order to verify the present study, a comparison of the thermal effect on the dimensionless natural frequency of the system for the first three modes with the results found in the literature is given in the tabular form.

Because of the strong coupling between mechanical and electrical phenomenon in electromechanical microdevices, there is a growing need for results with temperature effect since they can give contribution to the making of modern microsensors. The ability of the MEMS device is precision and sensitivity without the need for any cumbersome electrical components.

\section{FORMULATION}

On the basis of the MCST, we discuss the oscillatory system of two parallel EulerBernoulli microbeams which are continuously joined by the Pasternak elastic layer under the influence of axial loading including the temperature change effect (see Fig. 1).The Pasternak foundation assumes the presence of shear interaction among the spring elements which is achieved by connecting the ends of the springs to a beam that only undergoes transverse shear deformation, see [25]. The load-deflection relationship is obtained by taking into account the vertical equilibrium of a shear layer. The pressure-deflection relationship is given by

$$
p=k w_{0 i}-G \frac{\partial^{2} w_{0 i}}{\partial x^{2}}, \quad i=1,2,
$$

where $G$ is the modulus of a shear Pasternak's layer and $K$ is the stiffness modulus of a Winkler elastic layer. Beams are continuously connected with the Winkler elastic layer which represents an idealized medium formed of system springs. Both the microbeams are rectangular and have the same length $L$, thickness $h$, width $b$.

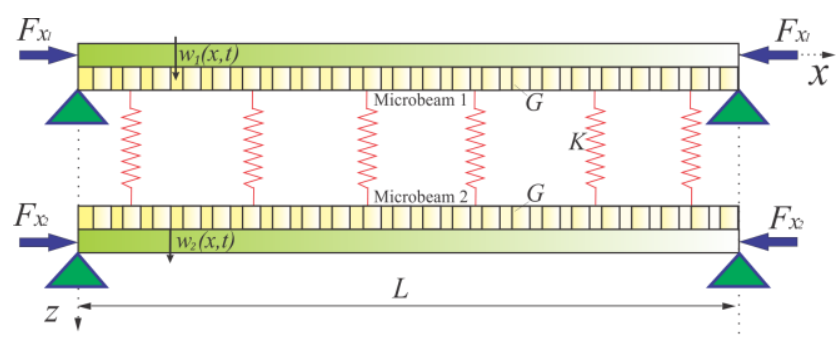

Fig. 1 Double-microbeam system coupled by the Pasternak's layer 
The beams are simply supported at the ends and under the effect of the axial compressive load with the temperature change effect.

\subsection{Introduction of the modified coupled stress theory}

The MCST was developed from the classical couple stress theory, which was well grounded by Mindlin [26], Mindlin and Tiersten [27], Toupin [28] and Koiter [29]. This theory, suggested by Yang et al.(2002), holds that energy density is a function of strain as well as curvature. According to the modified couple stress theory, Yang [30], Park and Gao [18], Ma et al. [19] and Reddy [17], strain energy $U_{s}$ in an isotropic linear elastic material occupying area $\Omega$ with a volume element $V$, and, can be written as

$$
U_{s}=\frac{1}{2} \int_{\Omega}(\sigma: \varepsilon+\boldsymbol{m}: \chi) d V,
$$

where $\sigma$ and $\varepsilon$ are the Cauchy stress tensor and strain tensor, respectively, $\boldsymbol{m}$ is the deviatory part of the couple stress tensor, and $\chi$ is the symmetric part of the curvature tensor. These tensors correspond to the geometrical equations:

$$
\varepsilon=\frac{1}{2}\left[\nabla \boldsymbol{u}+(\nabla \boldsymbol{u})^{T}\right], \chi=\frac{1}{2}\left[\nabla \boldsymbol{\theta}+(\nabla \boldsymbol{\theta})^{T}\right], \nabla=\left[\begin{array}{lll}
\frac{\partial}{\partial x} & \frac{\partial}{\partial y} & \frac{\partial}{\partial z}
\end{array}\right],
$$

where $\nabla$ is the Nabla operator, $\boldsymbol{u}$ is the displacement vector. The rotation vector and constitutive equations are defined by

$$
\begin{gathered}
\boldsymbol{\theta}=\frac{1}{2} \text { curlu }, \\
\boldsymbol{\sigma}=\lambda \operatorname{tr}(\boldsymbol{\varepsilon}) \boldsymbol{I}+2 \mu \boldsymbol{\varepsilon}, \boldsymbol{m}=2 l^{2} \mu \chi .
\end{gathered}
$$

Where material length scale parameter $l$ has the dimension of length which is mathematically the square of the ratio of the curvature modulus to the shear modulus and is physically regarded as a material property measuring the effect of couple stress, Mindlin [26], $\mu$ and $\lambda$ are the Lamé constants that are given as:

$$
\lambda=\frac{E v}{(1+v)(1-2 v)} \text { and } \mu=\frac{E}{2(1+v)} .
$$

In order to implement the linear constitutive relations presented in Eq. (6) the microbeam material should be made homogeneous, isotropic and linearly elastic.

\subsection{Mathematical model of the double-microbeam system}

Based on the Euler-Bernoulli beam theory, axial displacements $u(x, z, t)$ and transverse displacements of any point of the beam, $w(x, z, t)$ are given by Reddy [14] as

$$
u(x, z, t)=-z \frac{\partial W_{0}(x, t)}{\partial x}, v(x, z, t)=0, w(x, z, t)=W_{0}(x, t),
$$


where $W_{0}(x, t)$ is the midplane displacement. From vector Eqs. (3)-(5) and displacements (7) it follows that

$$
\begin{gathered}
\varepsilon_{x x}=-z \frac{\partial^{2} W_{0}(x, t)}{\partial x^{2}}, \varepsilon_{y y}=\varepsilon_{z z}=\varepsilon_{x z}=\varepsilon_{y z}=\varepsilon_{x y}=0, \theta_{y}=-\frac{\partial W_{0}(x, t)}{\partial x}, \theta_{x}=\theta_{z}=0, \\
\chi_{x y}=-\frac{1}{2} \frac{\partial^{2} W_{0}(x, t)}{\partial x^{2}}, \chi_{y y}=\chi_{z z}=\chi_{x z}=\chi_{y z}=\chi_{x y}=0, \\
m_{x y}=-\mu l^{2} \frac{\partial^{2} W_{0}(x, t)}{\partial x^{2}}, m_{x x}=m_{y y}=m_{z z}=m_{x z}=m_{y z}=0, \\
\sigma_{x x}=-\frac{E(1-v)}{(1+v)(1-2 v)}\left(z \frac{\partial^{2} W_{0}(x, t)}{\partial x^{2}}+\alpha \Delta T\right), \sigma_{y y}=\sigma_{z z}=\sigma_{x z}=\sigma_{y z}=\sigma_{x y}=0,
\end{gathered}
$$

where $E$ is Young's modulus, $\alpha$ is the coefficient of thermal expansion, $v$ is Poisson's ratio and $\Delta T=T-T_{0}$ is the temperature change with a respect to reference temperature $T_{0}$ and assuming no shear strains are created by temperature change.

In this study, the equilibrium equations are derived by the principle of total potential energy $[15,16]$. From Eqs (2) and (8) - (11), the variation of strain energy in the doublebeam system can be determined as

$$
\delta U_{s}=\int_{\Omega}\left(\sigma_{x x} \delta \varepsilon_{x x}+2 m_{x y} \delta \chi_{x y}\right) d V=\int_{0}^{L}\left(-M_{x i} \delta \frac{\partial^{2} W_{0 i}}{\partial x^{2}}-Y_{x y i} \delta \frac{\partial^{2} W_{0 i}}{\partial x^{2}}\right) d x,(i=1,2),
$$

where $M_{x i}$ and $Y_{x y i}$ are the stress resultant moments and couple moments for the first and second microbeam, respectively, defined as

$$
M_{x i}=\int_{A_{i}} z \sigma_{x x} d A_{i}=-D_{x x} \frac{\partial^{2} W_{0 i}}{\partial x^{2}}, \quad Y_{x y i}=\int_{A_{i}} m_{x y} d A_{i}=-A_{x z} l^{2} \frac{\partial^{2} W_{0 i}}{\partial x^{2}} .
$$

The stiffness components in Eqs. (13) are defined as [22]

$$
\left\{A_{x x}, D_{x x}\right\}=\int_{A_{i}} \frac{E_{i}(1-v)}{(1+v)(1-2 v)}\left\{1, z^{2}\right\} d A_{i}, A_{x z}=\int_{A_{i}} \mu d A_{i},(i=1,2) .
$$

Using the displacement field components given in Eq. (7), we obtain the variation of kinetic energy in the form

$$
\delta K_{e}=-\int_{0}^{L} m_{0 i} \frac{\partial^{2} W_{0 i}}{\partial t^{2}} \delta W_{0 i} d x, m_{0 i}=\int_{A_{i}} \rho_{i} d A_{i}=\rho_{i} A_{i},(i=1,2),
$$

where $\rho_{i}$ is the mass density for the first and second microbeam. The first variation of the additional strain energy caused by the elastic medium is written by

$$
\delta U_{a d}=\int_{0}^{L}\left[K\left(W_{01}-W_{02}\right) \delta\left(W_{01}-W_{02}\right)-G \frac{\partial W_{01}}{\partial x} \delta \frac{\partial W_{01}}{\partial x}-G \frac{\partial W_{02}}{\partial x} \delta \frac{\partial W_{02}}{\partial x}\right] d x
$$


where $K$ and $G$ are the spring constants of the Winkler and Pasternak elastic medium, respectively. The first variation of the work done by axial forces $F_{x i}=F_{m i}+F_{T}$, $(i=1,2)$ can be given as

$$
\delta W_{e x t}=\int_{0}^{L}\left(F_{m i}+F_{T}\right) \frac{\partial W_{0 i}}{\partial x} \delta \frac{\partial W_{0 i}}{\partial x} d x,(i=1,2),
$$

where $F_{T}=A_{x x} \alpha \Delta T$ is the axial force due to the influence of the temperature change and $F_{m i},(i=1,2)$ is the axial forces due to the mechanical loading for the first and second microbeams. The main equation and the boundary conditions can be derived by the Hamilton principles as follows

$$
\int_{0}^{t}\left[\delta K_{e}-\left(\delta U_{s}+\delta U_{a d}+\delta W_{e x t}\right)\right] d t=0 .
$$

If we substitute the expressions for $\delta U_{s}, \delta K_{e}, \delta U_{a d}$ and $\delta W_{e x t}$ from Eqs. (12), (15), (16) and (17) into Eq. (18) and after integrating by parts and then collecting the coefficients of $\delta W_{01}$ and $\delta W_{02}$, the equations of motion of the double microbeam system are obtained in the form

$$
\begin{gathered}
\delta W_{01}: m_{01} \frac{\partial^{2} W_{01}}{\partial t^{2}}=\frac{\partial^{2} M_{x 1}}{\partial x^{2}}+\frac{\partial Y_{x y 1}}{\partial x^{2}}-\left(F_{m 1}+F_{T}\right) \frac{\partial^{2} W_{01}}{\partial x^{2}}+G \frac{\partial^{2} W_{01}}{\partial x^{2}}-K\left(W_{01}-W_{02}\right), \\
\delta W_{02}: m_{02} \frac{\partial^{2} W_{02}}{\partial t^{2}}=\frac{\partial^{2} M_{x 2}}{\partial x^{2}}+\frac{\partial Y_{x y 2}}{\partial x^{2}}-\left(F_{m 2}+F_{T}\right) \frac{\partial^{2} W_{02}}{\partial x^{2}}+G \frac{\partial^{2} W_{02}}{\partial x^{2}}+K\left(W_{01}-W_{02}\right) .
\end{gathered}
$$

The boundary and initial conditions of the double-microbeam system are assumed to be simply supported and considered as

$$
\begin{gathered}
W_{0 i}(0, t)=W_{0 i}(L, t)=0, \frac{\partial^{2} W_{0 i}}{\partial x^{2}}(0, t)=\frac{\partial^{2} W_{0 i}}{\partial x^{2}}(L, t)=0,(i=1,2), \\
W_{0 i}(x, 0)=f_{i}(x), \frac{\partial W_{0 i}}{\partial t}(x, 0)=g_{i}(x),(i=1,2) .
\end{gathered}
$$

\section{ANALYTICAL SOLUTION PROCEDURE}

For the sake of simplicity, we assume that the two parallel beams of the elastically connected double-beam system have the same bending stiffness $E I_{1}=E I_{2}=E I$ and crosssectional area $A_{1}=A_{2}=A$. Both microbeams have the same length $L$ and same material characteristics $\rho_{1}=\rho_{2}=\rho$. The equations of motion can be expressed in the terms of displacements $W_{01}$ and $W_{02}$. By substituting Eqs. (14) into Eqs. (19) and (20) the main equations of EBDMBS in terms of the displacements are given below

$$
\left(D_{x x}+A_{x z} l^{2}\right) \frac{\partial^{4} W_{01}}{\partial x^{4}}+m_{0} \frac{\partial^{2} W_{01}}{\partial t^{2}}+\left(F_{m 1}+F_{T}\right) \frac{\partial^{2} W_{01}}{\partial x^{2}}-G \frac{\partial^{2} W_{01}}{\partial x^{2}}+K\left(W_{01}-W_{02}\right)=0,
$$




$$
\left(D_{x x}+A_{x z} l^{2}\right) \frac{\partial^{4} W_{02}}{\partial x^{4}}+m_{0} \frac{\partial^{2} W_{02}}{\partial t^{2}}+\left(F_{m 2}+F_{T}\right) \frac{\partial^{2} W_{02}}{\partial x^{2}}-G \frac{\partial^{2} W_{02}}{\partial x^{2}}-K\left(W_{01}-W_{02}\right)=0 .
$$

In order to simplify the solving of Eqs. (23) and (24), we will introduce the following dimensionless parameters:

$$
\begin{aligned}
& w_{0 i}=\frac{W_{0 i}}{L}, \xi=\frac{x}{L}, l_{0}=\frac{l}{L}, \tau=\frac{t}{L} \sqrt{\frac{A_{x x}}{m_{0}}}, k_{w}=\frac{K L^{2}}{A_{x x}}, k_{p}=\frac{G}{A_{x x}}, \\
& \bar{F}_{T}=\frac{F_{T}}{A_{x x}}, \bar{F}_{m i}=\frac{F_{m i}}{A_{x x}}, d_{x x}=\frac{D_{x x}}{A_{x x}}, a_{x z}=\frac{A_{x z}}{A_{x x}}, \quad(i=1,2) .
\end{aligned}
$$

Assuming time harmonic motion and using separation of variables, the solutions of Eqs. (23) and (24) with the main boundary conditions (21) can be written in the form

$$
w_{0 i}(\xi, \tau)=\sum_{n=1}^{\infty} X_{n}(\xi) S_{i n}(\tau), X_{n}(\xi)=\sin \left(k_{n} \xi\right), k_{n}=n \pi,(i=1,2),
$$

where $S_{i n}(\tau)$ is the unknown time function, and $X_{n}(\xi)$ is the known mode shape function for a simply supported single microbeam. Introducing the general solutions (26) into Eqs. (23) and (24) we obtain the following equations

$$
\begin{aligned}
& \ddot{S}_{1 n}+\left[\left(d_{x x}+a_{x z} l_{0}^{2}\right) k_{n}^{4}-\left(\bar{F}_{m 1}+\bar{F}_{T}\right) k_{n}^{2}+k_{p} k_{n}^{2}+k_{w}\right] S_{1 n}-k_{w} S_{2 n}=0, \\
& \ddot{S}_{2 n}+\left[\left(d_{x x}+a_{x z} l_{0}^{2}\right) k_{n}^{4}-\left(\bar{F}_{m 2}+\bar{F}_{T}\right) k_{n}^{2}+k_{p} k_{n}^{2}+k_{w}\right] S_{2 n}-k_{w} S_{1 n}=0,
\end{aligned}
$$

The solutions of Eqs. (27) and (28) are assumed in the following forms

$$
S_{1 n}=C_{n} e^{j \omega_{n} \tau}, \quad S_{2 n}=D_{n} e^{j \omega_{n} \tau}, \quad j=\sqrt{-1},
$$

where $\omega_{n}$ marks the natural frequency of the double-microbeam system, and $C_{n}$ and $D_{n}$ present the amplitude coefficients of the two microbeams, respectively. By substituting Eqs. (29) into Eqs. (27) and (28) the determinant can be written from which the nontrivial solutions for constants $C_{n}$ and $D_{n}$ can be obtained only when this determinant of the coefficients vanishes. This gives the following frequency equations

$$
\begin{gathered}
\omega_{n}^{4}-\left[2\left(d_{x x}+a_{x z} l_{0}^{2}\right) k_{n}^{4}-\left(\bar{F}_{m 1}+\bar{F}_{T}\right) k_{n}^{2}-\left(\bar{F}_{m 2}+\bar{F}_{T}\right) k_{n}^{2}+2 k_{p} k_{n}^{2}+2 k_{w}\right] \omega_{n}^{2}+ \\
{\left[\left(d_{x x}+a_{x z} l_{0}^{2}\right) k_{n}^{4}-\left(\bar{F}_{m 1}+\bar{F}_{T}\right) k_{n}^{2}+k_{p} k_{n}^{2}+k_{w}\right]\left[\left(d_{x x}+a_{x z} l_{0}^{2}\right) k_{n}^{4}-\left(\bar{F}_{m 2}+\bar{F}_{T}\right) k_{n}^{2}+k_{p} k_{n}^{2}+k_{w}\right]} \\
-k_{w}^{2}=0 .
\end{gathered}
$$

Finally, when the bi-axial compression forces due to the mechanical loading $\bar{F}_{m 1}=\bar{F}_{m 2}$ $=0$ are ignored, the natural frequency of the system is written by the formula

$$
\left(\omega_{n I, I I}^{0}\right)^{2}=\left(d_{x x}+a_{x z} l_{0}^{2}\right) k_{n}^{4}-\bar{F}_{T} k_{n}^{2}+k_{p} k_{n}^{2}+k_{w} \mp k_{w},
$$

where $\omega_{n I}^{0}$ is the lower natural frequency and $\omega_{n I I}^{0}$ is the higher natural frequency of the EBDMBS. When the bi-axial load applied on the double-beam system reaches a certain critical 
value, the double-beam system becomes unstable which means that the system begins to buckle. Introducing $\omega_{n}=0$ into Eq. (30), and substituting mechanical load ratio $\vartheta=\bar{F}_{m 2} / \bar{F}_{m 1}$, where $\bar{F}_{m 1}=F$ and $\bar{F}_{m 2}=\vartheta F$, we obtain the equation for the critical buckling load as follows

$$
\begin{gathered}
F_{c r}=\frac{1}{2 \vartheta k_{n}^{4}}\left\{\left[\left(d_{x x}+a_{x z} l_{0}^{2}\right) k_{n}^{4}-\bar{F}_{T} k_{n}^{2}+k_{p} k_{n}^{2}+k_{w}\right](1+\vartheta) k_{n}^{2}-\sqrt{b_{1}^{2}-4 \vartheta k_{n}^{4} c_{1}}\right\}, \\
b_{1}=\left[\left(d_{x x}+a_{x z} l_{0}^{2}\right) k_{n}^{4}-\bar{F}_{T} k_{n}^{2}+k_{p} k_{n}^{2}+k_{w}\right](1+\vartheta) k_{n}^{2}, \\
c_{1}=\left[\left(d_{x x}+a_{x z} l_{0}^{2}\right) k_{n}^{4}-\bar{F}_{T} k_{n}^{2}+k_{p} k_{n}^{2}+k_{w}\right]^{2}-k_{w}^{2} .
\end{gathered}
$$

Based on equation (32), the critical buckling temperature of the EBDMBS for the biaxial compression equal to zero $\bar{F}_{m 1}=\bar{F}_{m 2}=0$ is of the form

$$
\left(\Delta T_{c r}\right)_{M C S T}=\frac{1}{\alpha A_{x x}}\left[\left(d_{x x}+a_{x z} l_{0}^{2}\right) k_{n}^{2}+k_{p}\right] .
$$

The illustrated analytical expressions for the natural frequency equation (31), critical buckling load equation (32) and the critical buckling temperature (35) are common equations for the EBDMBS with thermal influence.

\subsection{Out-of-phase modes of vibration and buckling state}

A detailed analysis for different cases of phase modes of vibration and buckling state is shown in the paper of Murmu and Adhikari [33, 34, 35].

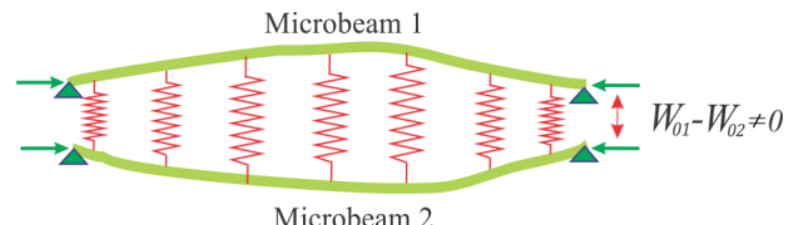

Fig. 2 Out-of-phase vibration of the double-microbeam system

For the EBDMBS we can use a change in variables by considering $W_{0 i}(x, t)$ as the relative displacement of the microbeam-1 with respect to the microbeam- 2

$$
W_{0}=W_{01}-W_{02},
$$

then

$$
W_{01}=W_{0}+W_{02} .
$$

Subtracting Eq. (23) from Eq. (22) and using Eqs. (36) and (37) we obtain

$$
\left(D_{x x}+A_{x z} l^{2}\right) \frac{\partial^{4} W_{0}}{\partial x^{4}}+m_{0} \frac{\partial^{2} W_{0}}{\partial t^{2}}+\left(F_{m}+F_{T}\right) \frac{\partial^{2} W_{0}}{\partial x^{2}}-G \frac{\partial^{2} W_{0}}{\partial x^{2}}+2 K W_{0}=0,
$$




$$
\left(D_{x x}+A_{x z} l^{2}\right) \frac{\partial^{4} W_{02}}{\partial x^{4}}+m_{0} \frac{\partial^{2} W_{02}}{\partial t^{2}}+\left(F_{m}+F_{T}\right) \frac{\partial^{2} W_{02}}{\partial x^{2}}-G \frac{\partial^{2} W_{02}}{\partial x^{2}}=K W_{0} .
$$

In above Eqs. (38) and (39) for sake of simplicity we assume that $F_{m 1}=F_{m 2}=F_{m}$.

If material length scale parameter $l$ is ignored, the above equations become those of the classical Euler-Bernoulli beam theory.

For the present out-of-phase analysis of the EBDMBS, we see simplicity in using Eq. (38). The general solution of Eq. (38) is written as

$$
W_{0}=\overline{W_{0}}(x) e^{i \omega t}, \quad i=\sqrt{-1},
$$

where $W_{0}(x)$ is the corresponding deformation shape of the EBDMBS and $\omega$ is frequency. For vibration analysis we know that is $F_{m}=0$. By introducing Eq. (40) in Eq. (38) we get

or

$$
\left(D_{x x}+A_{x z} l^{2}\right) \frac{\partial^{4} \overline{W_{0}}(x)}{\partial x^{4}}+\left(F_{T}-G\right) \frac{\partial^{2} \overline{W_{0}}(x)}{\partial x^{2}}-\left(m_{0} \omega^{2}-2 K\right) \overline{W_{0}}(x)=0,
$$

$$
A_{1} \frac{\partial^{4} \overline{W_{0}}(x)}{\partial x^{4}}+A_{2} \frac{\partial^{2} \overline{W_{0}}(x)}{\partial x^{2}}-A_{3} \overline{W_{0}}(x)=0
$$

where the coefficients are

$$
\left(D_{x x}+A_{x z} l^{2}\right)=A_{1},\left(F_{T}-G\right) A_{2},\left(m_{0} \omega^{2}-2 K\right)=A_{3} .
$$

The general solution of Eq. (42) can be written as

$$
\bar{W}_{0}(x)=C_{1} \sin \lambda_{1} x+C_{2} \cos \lambda_{1} x+C_{3} \sinh \lambda_{2} x+C_{4} \cosh \lambda_{2} x,
$$

where $C_{k},(k=1,2,3,4)$ can be determined from the boundary conditions (21) and

$$
\lambda_{1}^{2}=\frac{1}{2 A_{1}}\left(A_{2}+\sqrt{A_{2}^{2}+4 A_{1} A_{3}}\right), \lambda_{2}^{2}=\frac{1}{2 A_{1}}\left(A_{2}-\sqrt{A_{2}^{2}+4 A_{1} A_{3}}\right) .
$$

Further, the solving of frequency for the out-of-phase vibration is presented in this section. The configuration of the EBDMBS with out-of-phase vibration mode $\left(W_{01}-W_{02} \neq 0\right)$ is shown in Fig. 2. By using the boundary conditions of simply-supported microbeam system from Eq. (21) yields $C_{2}=0$ and $C_{4}=0$.

From that we can write

$$
\left[\begin{array}{cc}
\sin \lambda_{1} L & \sinh \lambda_{2} L \\
\left(D_{x x}+A_{x z} l^{2}\right) \lambda_{1}^{2} \sin \lambda_{1} L & \left(D_{x x}+A_{x z} l^{2}\right) \lambda_{2}^{2} \sinh \lambda_{2} L
\end{array}\right]\left\{\begin{array}{l}
C_{1} \\
C_{3}
\end{array}\right\}=\left\{\begin{array}{l}
0 \\
0
\end{array}\right\} .
$$

For the nontrivial solution of Eq. (46) the determinant is zero, it follows

$$
\sin \lambda_{1} L\left[\left(D_{x x}+A_{x z} l^{2}\right) \lambda_{2}^{2} \sinh \lambda_{2} L-\left(D_{x x}+A_{x z} l^{2}\right) \lambda_{1}^{2} \sinh \lambda_{2} L\right]=0 .
$$

From Eq. (47) the frequency equation is

$$
\sin \lambda_{1} L=0
$$


and implies

Using Eq. (45) yields

$$
\lambda_{1} L=n \pi, \quad n=1,2 \ldots
$$

$$
A_{1} \lambda_{1}^{4}-A_{2} \lambda_{1}^{2}-A_{3}=0
$$

Using the dimensionless parameters (25) and Eq. (43), the natural frequency of the EBDMBS for out-of-phase vibration mode we get

$$
\Omega_{n}=\sqrt{\left(d_{x x}+a_{x z} l_{0}^{2}\right) k_{n}^{4}-\left(\bar{F}_{T}-k_{p}\right) k_{n}^{2}+2 k_{w}} .
$$

Using dimensionless parameters (25) and Eq. (38) we get the expression of buckling load in out-of-phase sequence as

$$
F_{n}=\frac{\left(d_{x x}+a_{x z} l^{2}\right) k_{n}^{4}-\left(\bar{F}_{T}-k_{p}\right) k_{n}^{2}+2 k_{w}}{k_{n}^{2}},
$$

\subsection{In-phase modes of vibration and buckling state}

The configuration of the EBDMBS with in-phase modes of vibration is shown in Fig. 3. The relative displacements between the two microbeams are absent $\left(W_{01}-W_{02}=0\right)$. For the mentioned EBDMBS vibration we solve the Eq. (39).

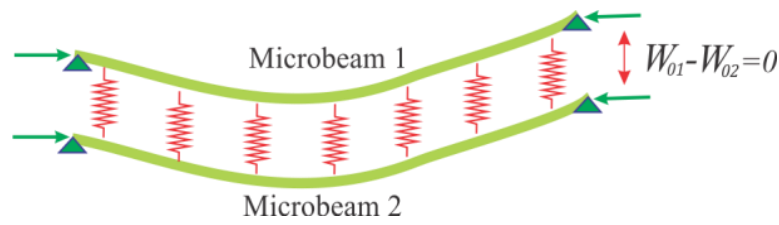

Fig. 3 Out-of-phase vibration of the double-microbeam system

By applying the same procedure from the previous chapter the natural frequencies of the EBDMBS for in-phase vibration mode can be expressed as

$$
\Omega_{n}=\sqrt{\left(d_{x x}+a_{x z} l_{0}^{2}\right) k_{n}^{4}-\left(\bar{F}_{T}-k_{p}\right) k_{n}^{2}} .
$$

The microbeams are buckled in the same direction (synchronous), see Fig. 3.Using dimensionless parameters (25) and Eq. (39) we get the expression of buckling load in inphase sequence as

$$
F_{n}=\left(d_{x x}+a_{x z} l^{2}\right) k_{n}^{2}-\left(\bar{F}_{T}-k_{p}\right) .
$$

It is shown from Eqs. (53) and (54) that the in-phase vibration mode and buckling state of the EBDMBS is independent of the stiffness of the connecting springs while it is dependent on Pasternak's layer and temperature effect and hence the EBDMBS can be treated as a single microbeam. A similar analysis for nanobeam system is presented in the paper of Murmu and Adhikari [33, 35]. 


\section{NUMERICAL RESULTS AND DISCUSSION}

In this section, we have illustrated a comparative study of the analytical results written in this paper and the results found in the literature. The microbeams of the system are made of epoxy with the following properties: $v=0.38, \rho=1220 \mathrm{~kg} / \mathrm{m}^{3}, E=1.44 \mathrm{GPa}$, $l=17.6 \mu \mathrm{m}, \alpha=54 \times 10^{-6} /{ }^{\circ} \mathrm{C}$ from [24]. The cross-section shape and length are kept the same by letting $b / h=2$ and $L / h=10$ respectively. Temperature and material length scale parameter effect on the two different cases of phase vibration modes and buckling state will be presented. A detailed parametric study is carried out by investigating the influence of different parameters on the natural frequency, frequency under the compressive axial loading, critical buckling load and critical temperature of the EBDMBS with thermal effect.

\subsection{Temperature and material length scale parameter effect on the phase vibration modes and phase buckling of the EBDMBS}

The frequency results of the EBDMBS are presented in terms of the frequency parameters for out-of-phase in Eq. (51) and in phase vibration mode in Eq. (53).Variation in frequency parameter $\Omega_{n}$ with material length scale parameter $l_{0}$, for different phase vibration due to temperature change is shown in Fig. 4. For the Winkler and Pasternak parameter we used constant values of $k_{w}=10$ and $k_{p}=0.1$, while for the temperature change we used two different values $\Delta T=50^{\circ} \mathrm{C}$ and $\Delta T=100^{\circ} \mathrm{C}$. It can be noticed from Fig. 4 that with increasing material length scale parameter $l_{0}$, frequency parameter $\Omega_{n}$ also increases for both considered cases of phase vibration. Frequency parameter $\Omega_{n}$ decreases as the temperature effect increases for both considered cases of phase vibration.

The buckling state results of the EBDMBS are presented in terms of the buckling parameters for out-of-phase in Eq. (52) and in phase buckling in Eq. (54). Material length scale parameter $\left(l_{0}\right)$ on buckling parameter $F_{n}$ for the different phase buckling due to temperature change is shown in Fig. 5. Also, it can be seen that as material length scale parameter $l_{0}$ increases, buckling parameter $F_{n}$ also increases for both considered cases of phase buckling. Buckling parameter $F_{n}$ decreases as the temperature effect increases for both considered cases of phase buckling.

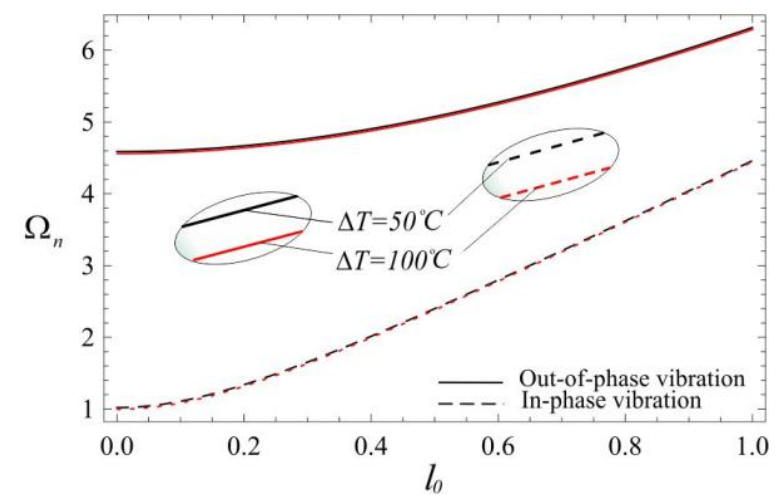

Fig. 4 Variation in frequency parameter $\Omega_{n}$ with material length scale parameter $\left(l_{0}\right)$ for different phase vibrations due to temperature change 


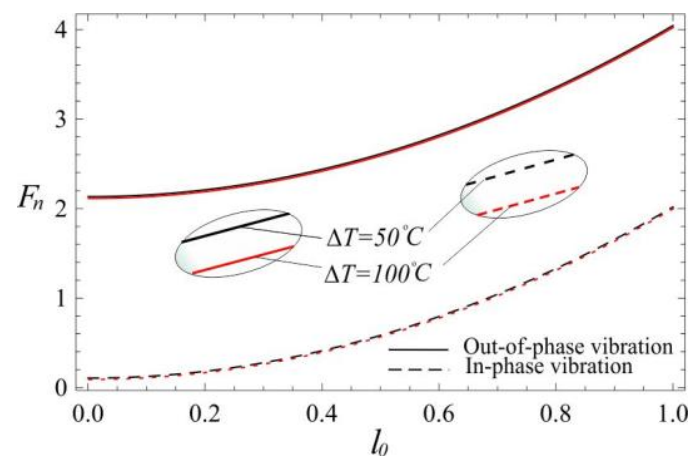

Fig. 5 Material length scale parameter $\left(l_{0}\right)$ on buckling parameter $F_{n}$ for the different phase buckling due to temperature change

\subsection{Thermal effect on the natural frequency of the EBDMBS}

It is commonly known that the lowest natural frequency and buckling load of systems of a larger number of coupled nano/micro structures correspond to the natural frequency and buckling load of one beam or plate, see Karličić et al. [31]. In this paper, the results for the lowest natural frequency and critical buckling load of the EBDMBS may be compared with those obtained for a microbeam one, presented in Ke et al. [24] and Ma et al. [19]. In order to confirm the present analytical method, Table 1 shows a comparison of thermal effect on the dimensionless natural frequency of the system from Eq. (31) for the first three modes with results Ke et al [24]. Perfect agreement between the present frequencies and those of Ke et al. [24] can be observed from Table 1. It is shown that the inclusion of the thermal effect decreases the frequencies of the microbeam one. It is seen that the effect of Pasternak parameter $k_{p}=0.01$, for a greater mode, leads to an increase in natural frequencies.

Table 1 Thermal effect on the dimensionless natural frequencies for the three modes of the microbeams with $h / l=2$

\begin{tabular}{|c|c|c|c|c|c|c|c|c|c|}
\hline \multirow[b]{2}{*}{$\Delta T\left({ }^{\circ} \mathrm{C}\right)$} & $n=1$ & $n=2$ & $n=3$ & $n=1$ & $\begin{array}{l}\text { Mode } \\
n=2\end{array}$ & $n=3$ & $n=1$ & $n=2$ & $n=3$ \\
\hline & \multicolumn{3}{|c|}{ Ke et al.[24] } & \multicolumn{3}{|c|}{ Present study for $k_{p}=0$} & \multicolumn{3}{|c|}{ Present study for $k_{p}=0.01$} \\
\hline 0 & 0.3478 & 1.2890 & 2.6277 & 0.3582 & 1.4328 & 3.2238 & 0.4764 & 1.5645 & 3.3588 \\
\hline 20 & 0.3322 & 1.2727 & 2.6099 & 0.3429 & $1.41^{\circ}$ & 3.2089 & 0.4651 & 1.5508 & 3.3444 \\
\hline 40 & 0.3159 & 1.2562 & 2.5920 & 0.3271 & 1.4027 & 3.1939 & 0.4535 & 1.5370 & 3.3300 \\
\hline 60 & 0.2986 & 1.2394 & 2.5739 & 0.3104 & 1.3875 & 3.1788 & 0.4416 & 1.5231 & 3.3156 \\
\hline 80 & 804 & 1.2225 & 2.5558 & 0.2927 & 1.3719 & 3.1637 & 0.4294 & 1.5090 & 3.3011 \\
\hline 100 & 0.2608 & 1.2053 & 2.5374 & 0.2739 & 1.3563 & 3.1485 & 0.4168 & 1.4948 & 3.2866 \\
\hline
\end{tabular}

It can be seen from Fig. 6 that the natural frequency with Poisson's ratio (i.e. $v=0.38$ ), suggested by the present Euler-Bernoulli beam model is always higher than that by Poisson's ratio $v=0$. The similar results, merely for a Timoshenko beam, are presented by Ma et al. [19]. We can conclude that there is perfect agreement between the present frequencies and those of Ma et al. [19], when we ignore an effect of the elastic medium, i.e. $k_{p}=0$ and $k_{w}=0$. 
The temperature effect and the Pasternak's parameter on the natural frequency can also be noticed in Fig. 6. The natural frequency decreases with temperature effect, in this case $100^{\circ} \mathrm{C}$, for a given value of $h / l$. It is noticed that the inclusion of the constant values of Pasternak's parameter $\left(k_{p}=0.01\right)$ increases the natural frequency of the EBDMBS. As a significant result, Fig. 6 shows that the increase in bending rigidity is suggested by the present model. Also important is that the difference between the natural frequency with the Poisson's ratio and the one without it is important only when the beam thickness is too small.

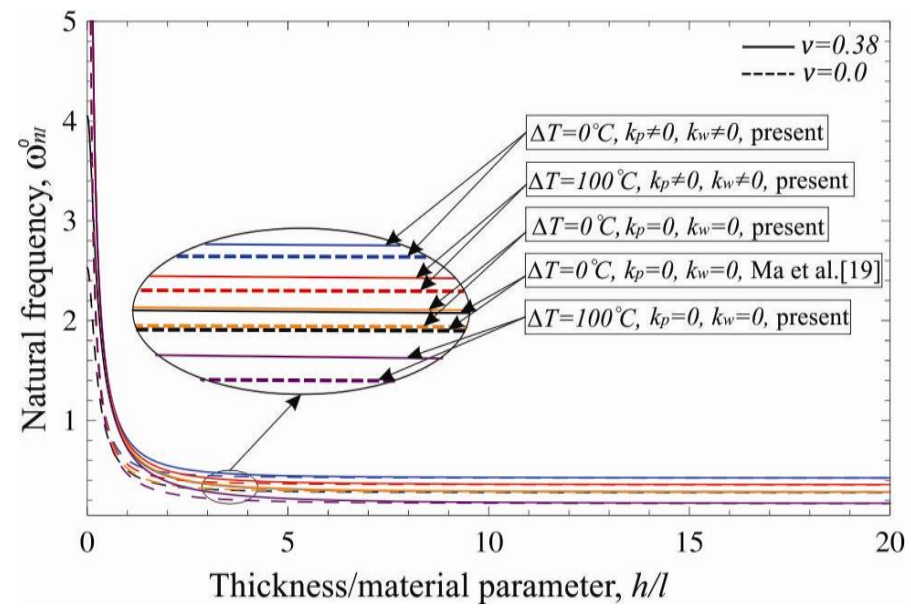

Fig. 6 The natural frequency of the EBDMBS varying with microbeam thickness, temperature effect and Pasternak's parameter

\subsection{The effect of the compression axial load and temperature effect on the EBDMBS}

To investigate the influence of the compressive axial loading on the natural frequencies of EBDMBS transverse vibration, we can compare the results of natural frequencies under the compressive axial loading and those without axial loading.

$$
\begin{gathered}
\omega_{n I, I I}^{2}=\frac{b_{2} \mp \sqrt{b_{2}^{2}-4 c_{2}}}{2}, \\
b_{2}=2\left(d_{x x}+a_{x z} l_{0}^{2}\right) k_{n}^{4}-\left(\phi F_{c r}+\bar{F}_{T}\right) k_{n}^{2}-\left(\phi \vartheta F_{c r}+\bar{F}_{T}\right) k_{n}^{2}+2 k_{p} k_{n}^{2}+2 k_{w}, \\
c_{2}=\left[\left(d_{x x}+a_{x z} l_{0}^{2}\right) k_{n}^{4}-\left(\phi F_{c r}+\bar{F}_{T}\right) k_{n}^{2}+k_{p} k_{n}^{2}+k_{w}\right] \\
{\left[\left(d_{x x}+a_{x z} l_{0}^{2}\right) k_{n}^{4}-\left(\phi \vartheta F_{c r}+\bar{F}_{T}\right) k_{n}^{2}+k_{p} k_{n}^{2}+k_{w}\right]-k_{w}^{2}, \quad \text { and } \phi=\frac{F_{m 1}}{F_{c r}} .}
\end{gathered}
$$

If we define

$$
\psi_{1}=\frac{\omega_{n I}}{\omega_{n I}^{0}}, \quad \psi_{2}=\frac{\omega_{n I I}}{\omega_{n I I}^{o}}
$$

with vibration mode number $n=1$ the impact of the compressive axial loading on the natural frequencies of transverse vibration of the EBDMBS presented by ratios of $\psi_{1}$ and 
$\psi_{2}$ are shown in Fig. 7. Fig. 7 shows that the ratios of frequencies $\psi_{1}$ and $\psi_{2}$ decrease with increasing axial compressive load $\phi$. It can be noticed that the effect of the compressive axial loading on lower natural frequency $\omega_{n I}$ is practically independent of axial compression ratio $\vartheta$, whereas on higher natural frequency $\omega_{n I I}$ it is dependent on it. For the Winkler and Pasternak parameter we used constant values of $k_{w}=10$ and $k_{p}=0.1$, while for the temperature change $\Delta T=50^{\circ} \mathrm{C}$. It can be noticed from Fig. 7(b) that as the axial compression ratio $\vartheta$ increases, the ratio of frequency $\psi_{2}$ decreases. Also, it can be seen that the axial compression ratio on the ratio of frequency $\psi_{2}$ is independent of axial compression ratio $\vartheta$ for small axial compressive load $\phi$, while it is significant for a large axial compressive load.

Fig. 8 shows the thermal effect and effect of the Pasternak parameter on critical buckling load $F_{c r}$ for the EBDMBS as a function of axial load ratio $\vartheta$. With the axial load ratio $\vartheta$ increase, the critical buckling load decreases. For a taken value of axial load ratio $\vartheta$, the critical buckling load of the EBDMBS decreases with an increase in temperature change. As can be seen, for the higher value of the Pasternak parameter of $k_{p}=0.1$, the critical buckling load has a noticeably higher value.

(a)

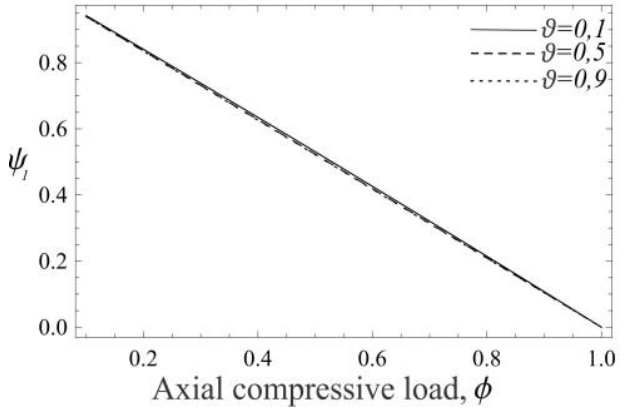

(b)

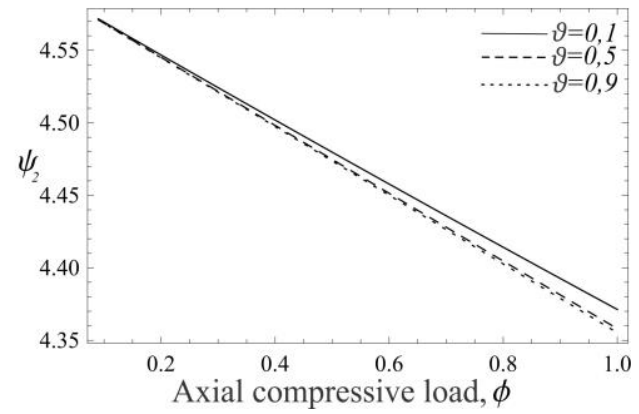

Fig. 7 The thermal effect on the relationships between ratios $\psi_{1}$ and $\psi_{2}$ and dimensionless parameter $\phi$ with increasing axial compressive load ratio $\vartheta$

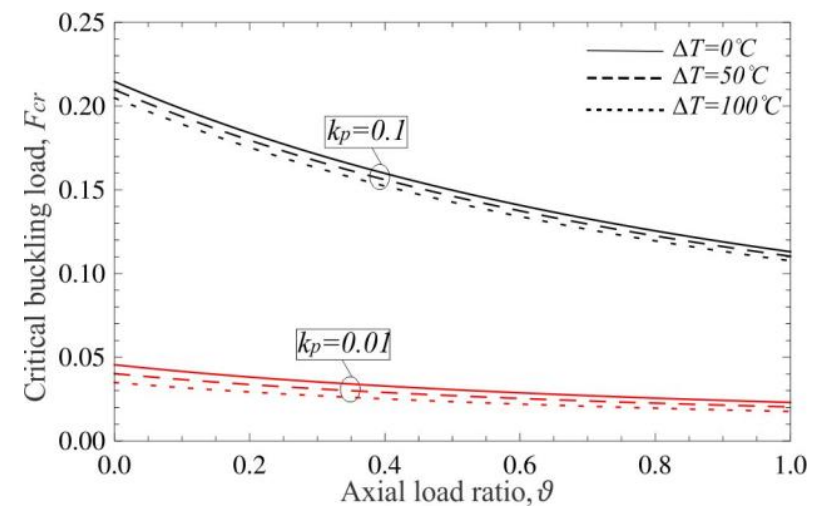

Fig. 8 The thermal effect and effect of Pasternak parameter on the critical buckling load $F_{c r}$ for the EBDMBS as a function of axial load ratio $\vartheta$ 
The critical scale load ratio of the modified and the local critical buckling loads at a low temperature environs is presented as

$$
T_{c r}=\frac{\Delta T_{c r M C S T}}{\Delta T_{\text {crClassical }}} .
$$

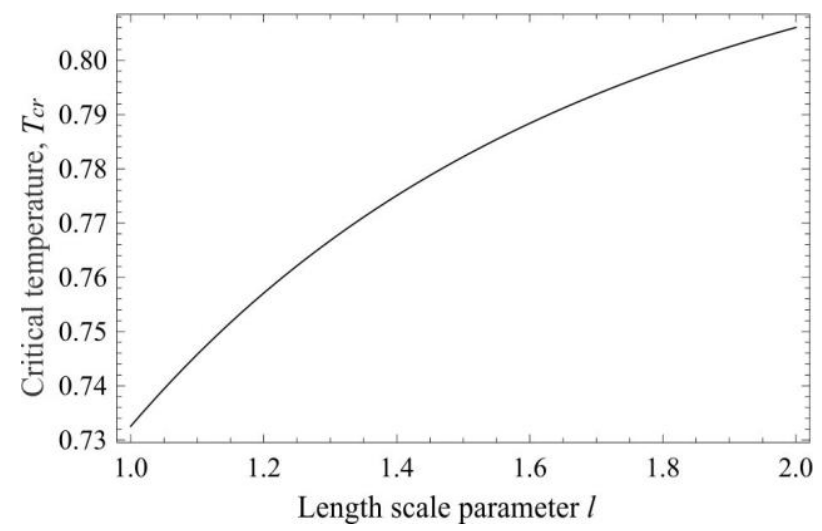

Fig. 9 Length scale parameter $T_{c r}$ at low temperature environs

In order to make a better illustration of the thermo-mechanical response of the EBDMBS, we introduced a scale parameter. Fig. 9 shows the influence of length scale parameter $T_{c r}$ at the low temperature environs. The influence of nonlocal parameter at low temperature environs is shown in Karličić et al. [32]. It can be observed from Fig. 9 that this parameter increases for a length scale parameter increase.

\section{CONCLUSIONS}

The thermal effect on the free vibration and buckling of the Euler-Bernoulli doublemicrobeam system is examined in this paper based on the modified couple stress theory. The system is composed of two identical, parallel, simply-supported beams which are continuously joined by the Pasternak's elastic layer. The temperature change effect is assumed to have an influence on both microbeams. The higher-order main equations and boundary conditions are derived using the Hamilton principle. The separation of variables method (known as the Fourier method) is used for the main equations to obtain free vibration frequencies and critical buckling loads of the EBDMBS. The length scale parameter, temperature change effect, critical buckling load, thickness/material parameter, Pasternak's parameter and Poisson's effect are discussed in detail. Also, the effect of different mentioned parameters on the natural frequency, frequency under the compressive axial loading, critical buckling load and critical temperature of the EBDMBS with thermal effect are presented. Effect of the material length scale parameter and thermal effect on the two different cases of phase modes of vibration and buckling state are discussed. Based on the presented analysis we conclude that the in-phase vibration mode and buckling state of the EBDMBS is independent of the stiffness of the connecting springs while it is 
dependent on Pasternak's layer and temperature effect and hence the EBDMBS can be treated as a single microbeam.

In order to confirm the present study, we have shown in tabular form a comparison of thermal effect on the dimensionless natural frequency of the system for three modes with the results found in the literature.

It is concluded that the presented results are in perfect agreement with the results observed in Ke et al. [24]. It is shown that the inclusion of the thermal effect decreases the frequencies of the microbeam one. Also, the effect of Pasternak parameter $k_{p}$ for a greater mode leads to an increase in natural frequencies, but including the temperature change, the frequency is decreased and leads to the decreased stiffness of the system. The numerical results obtained for the natural frequency with Poisson's effect and suggested by the present Euler-Bernoulli beam model are always higher than those without Poisson's effect. The thermal effect on the natural frequency is very low for the microbeam one of the EBDMBS and with a small ratio of $h / l$, while it is significant for the microbeam with a large ratio of $h / l$. The impact of the compressive axial loading on the natural frequencies of the EBDMBS transverse vibration leads to the following observations:

- The temperature change effect has an impact on both microbeams.

- The lower and higher natural frequency under the compressive axial loading decrease with increasing axial compressive load and also decrease with a temperature change increase. The reason for that is that the thermal effect leads to the reduction in stiffness and such a behavior leads to the softening of the materials of the EBDMBS.

- The effect of the compressive axial loading on the lower natural frequency is almost independent of the axial compression ratio, whereas on the higher natural frequency it depends on it.

- For a higher value of the Pasternak parameter, the critical buckling load has a higher value which decreases with increasing temperature change.

- The critical buckling temperature for the presented systems is always lower than for the classical theories.

- The critical scale load ratio of the modified and the local critical buckling loads at the low temperature environs increases with the increasing length scale parameter.

All these observations can be useful for modern electromechanical systems. Physical views of this paper may be useful for the design and vibration analysis of microresonators and microsensors applications. We have shown that using the presented system with the temperature change leads to considerable changes in stiffness, i.e. the thermal effect leads to the reduction in stiffness and such a behavior leads to the softening of the materials of the EBDMBS.

Acknowledgements: This research is supported by the research grant of the Serbian Ministry of Science and Environmental Protection under the numbers OI 174001 and OI 174011. 


\section{REFERENCES}

1. Lam, D. C. C., Yang, F., Chong, A. C. M., Wang, J., Tong, P., 2003, Experiments and theory in strain gradient elasticity, Journal of the Mechanics and Physics of Solids, 51(8), pp. 1477-1508.

2. Gallacher, B. J., Burdess, J. S., Harish, K. M., 2006, A control scheme for a MEMS electrostatic resonant gyroscope excited using combined parametric excitation and harmonic forcing, Journal of Micromechanics and Microengineering, 16(2), 320.

3. Kacem, N., Baguet, S., Hentz, S., Dufour, R., 2011, Computational and quasi-analytical models for nonlinear vibrations of resonant MEMS and NEMS sensors, International Journal of Non-Linear Mechanics, 46(3), pp. 532-542.

4. Harish, K. M., Gallacher, B. J., Burdess, J. S., Neasham, J. A., 2009, Experimental investigation of parametric and externally forced motion in resonant MEMS sensors, Journal of Micromechanics and Microengineering, 19(1), 015021.

5. Magrab, E. B., 2012, Vibrations of Elastic Systems: With Applications to MEMS and NEMS, Vol. 184, Springer.

6. Ilic, B., Krylov, S., Bellan, L. M., Craighead, H. G., 2007, Dynamic characterization of nanoelectromechanical oscillators by atomic force microscopy, Journal of applied physics, 101(4), 044308.

7. Hasanyan, DJ., Batra RC., Harutyunyan S., 2008, Pull-in instabilities in functionally graded microthermoelectromechanical systems, J Thermal Stress, 31, pp1006-1021.

8. Rahaeifard, M., Kahrobaiyan, M. H., Ahmadian, M. T., 2009, Sensitivity analysis of atomic force microscope cantilever made of functionally graded materials, 3rd international conference on micro-and nanosystems (MNS3), San Diego (CA, USA), In: DETC 2009-86254.

9. Mindlin, R. D., Eshel, N. N., 1968, On first strain-gradient theories in linear elasticity, International Journal of Solids and Structures, 4(1), pp. 109-124.

10. Wang, B., Zhao, J., Zhou, S., 2010, A micro scale Timoshenko beam model based on strain gradient elasticity theory, European Journal of Mechanics-A/Solids, 29(4), pp. 591-599.

11. Lazopoulos, K. A., Lazopoulos, A. K., 2010, Bending and buckling of thin strain gradient elastic beams, European Journal of Mechanics-A/Solids, 29(5), pp. 837-843.

12. Peddieson, J., Buchanan, G. R., McNitt, R. P., 2003, Application of nonlocal continuum models to nanotechnology, International Journal of Engineering Science, 41(3), pp. 305-312.

13. Eringen, A. C., 1983, On differential equations of nonlocal elasticity and solutions of screw dislocation and surface waves, Journal of Applied Physics, 54(9), pp. 4703-4710.

14. Reddy, J. N., 2007, Nonlocal theories for buckling bending and vibration of nanobeams, International Journal of Engineering Science, 45, pp. 288-307.

15. Reddy, J. N., 2002, Energy principles and variational methods in applied mechanics, 2nd ed. New York: John Wiley and Sons.

16. Reddy, J. N., 2008, An introduction to continuum mechanics with applications, New York, Cambridge University Press.

17. Reddy, J. N., 2011, Microstructure-dependent couple stress theories of functionally graded beams, Journal of the Mechanics and Physics of Solids, 59(11), pp. 2382-2399.

18. Park, S. K., Gao, X. L., 2006, Bernoulli-Euler beam model based on a modified couple stress theory, Journal of Micromechanics and Microengineering, 16(11), 2355.

19. Ma, H. M., Gao, X. L., Reddy, J. N., 2008, A microstructure-dependent Timoshenko beam model based on a modified couple stress theory, Journal of the Mechanics and Physics of Solids, 56(12), pp. 3379-3391.

20. Nateghi, A., Salamat-talab, M., Rezapour, J., Daneshian, B., 2012, Size dependent buckling analysis of functionally graded micro beams based on modified couple stress theory, Applied Mathematical Modelling, 36(10), pp. 4971-4987.

21. Bekir, A., Civalek, Ö., 2011, Strain gradient elasticity and modified couple stress models for buckling analysis of axially loaded micro-scaled beams, International Journal of Engineering Science, 49(11), pp. 1268-1280.

22. Şimşek, M., Reddy, J. N., 2013, A unified higher order beam theory for buckling of a functionally graded microbeam embedded in elastic medium using modified couple stress theory, Composite Structures, 101, pp. 47-58.

23. Hendou, R. H., Mohammadi, A.K., 2014, Transient analysis of nonlinear Euler-Bernoulli micro-beam with thermoelastic damping, via nonlinear normal modes, Journal of Sound and Vibration, 333(23), pp. 6224-6236. 
24. Ke, L. L., Wang, Y. S., Wang, Z. D., 2011, Thermal effect on free vibration and buckling of size-dependent microbeams, Physica E: Low-dimensional Systems and Nanostructures, 43(7), pp. 1387-1393.

25. Dutta S. C., Roy R., 2002, A critical review on idealization and modeling for interaction among soilfoundation-structure system, Computers and Structures, 80(1), pp. 1579-159.

26. Mindlin, R. D., 1963, Influence of couple-stresses on stress concentrations. Experimental mechanics, 3(1), pp. $1-7$.

27. Mindlin, R. D., Tiersten, H. F., 1962, Effects of couple-stresses in linear elasticity, Archive for Rational Mechanics and Analysis, 11(1), pp. 415-448.

28. Toupin, R. A., 1962, Elastic materials with couple-stresses, Archive for Rational Mechanics and Analysis, 11(1), pp. 385-414.

29. Koiter, W. T., 1964, Couple-stresses in the theory of elasticity: I and II, Proceedings of the Koninklijke Nederlandse Akademie van Wetenschappen, B67, pp. 17-44.

30. Yang, F. A. C. M., Chong, A. C. M., Lam, D. C. C., Tong, P., 2002, Couple stress based strain gradient theory for elasticity. International Journal of Solids and Structures, 39(10), pp. 2731-2743.

31. Karličić, D., Kozić, P., Pavlović, R., 2014, Free transverse vibration of nonlocal viscoelastic orthotropic multinanoplate system (MNPS) embedded in a viscoelastic medium, Composite Structures, 115, pp. 89-99.

32. Karličić, D., Cajić, M., Kozić, P., Pavlović, I., 2015, Temperature effects on the vibration and stability behaviour of multi-layered graphene sheets embedded in an elastic medium, Composite Structures, 131, pp. 672-681.

33. Murmu, T., Adhikari, S., 2010, Nonlocal transverse vibration of double-nanobeam-systems, Journal of Applied Physics, 108(8), p. 083514.

34. Murmu, T., Adhikari, S., 2010, Nonlocal effects in the longitudinal vibration of double-nanorod systems, Physica E: Low-dimensional Systems and Nanostructures, 43(1), pp. 415-422.

35. Murmu, T., Adhikari, S., 2011, Axial instability of double-nanobeam-systems, Physics Letters A, 375(3), pp. 601-608. 